\title{
The compound Poisson distribution and return times in dynamical systems
}

\author{
Nicolai Haydn* Sandro Vaienti ${ }^{\dagger}$
}

October 29,2018

\begin{abstract}
Previously it has been shown that some classes of mixing dynamical systems have limiting return times distributions that are almost everywhere Poissonian. Here we study the behaviour of return times at periodic points and show that the limiting distribution is a compound Poissonian distribution. We also derive error terms for the convergence to the limiting distribution. We also prove a very general theorem that can be used to establish compound Poisson distributions in many other settings.
\end{abstract}

\section{Introduction}

In 1899 Poincaré showed that for a map $T$ on some space $\Omega$ which has an invariant probability measure $\mu$, almost every every point returns within finite time arbitrarily close. This means that for every (measurable) $A \subset \Omega$ with $\mu(A)>0$ the return time function $\tau_{A}(x)=\min \left\{k \geq 1: T^{k} x \in A\right\}$ is finite for $\mu$-almost every $x \in A$. This result was quantified by Kac in 1947 for ergodic measures. His theorem states that $\int_{A} \tau_{A}(x) d \mu(x)=1$, provided $\mu$ is ergodic, which implies that $\tau_{A}(x)$ is on average equal to $1 / \mu(A)$. Since 1990 there has been a growing interest in the statistics of return times and in particular in the distribution of $\tau_{A}$. Considering that it was shown in 20, 19] that for ergodic measures the limiting distribution of a sequence of (rescaled) return functions $\tau_{U_{n}}$ for $n \rightarrow \infty$ can be any arbitrarily prescribed distribution for suitably chosen sets $U_{n}$, it is necessary to assume that the return sets $A$ are dynamically regular.

For the measure of maximal entropy on a subshift of finite type, Pitskel [25] showed that the return times are in the limit Poisson distributed for cylinder sets $A_{n}(x)\left(\mu\left(A_{n}(x)\right) \rightarrow 0\right.$ as $\left.n \rightarrow \infty\right)$ where the set of suitable 'centres' $x \in \Omega$ form a full measure set. A similar result had independently been obtained by Hirata [15, 16] by a different method. For $\phi$-mixing Gibbs measures Galves and Schmitt [10] showed in 1997 that the first return time is in the limit exponentially distributed and that the convergence is at an exponential rate. Subsequent results (e.g. 27, 8, 17, 13, 5, 1]) established limiting distribution results for first or multiple return times in various settings and sometimes with rates of convergence.

Almost all previous results look at the distribution of return times near generic points. The notable exception being the paper [15] by Hirata which gives the distribution of the first return time at a periodic point. In the present paper we consider periodic points for sufficiently well mixing invariant measures and show that the limiting distribution is compound Poissonian. The compound Poisson distribution has previously been used in various settings including the analysis of internet traffic where the waiting time between packets is exponential and the size of each packet is geometrically distributed. It has also been used to model the survival of capitalist enterprises in the free market system 21. The main technical result, Proposition 1] provides conditions under which one obtains a compound Poissonian

*Mathematics Department, University of Southern California, Los Angeles, 90089-1113. E-mail: $<$ nhaydn@math.usc.edu $>$. This work was supported by a grant from the NSF (DMS-0301910) and by a grant from the Université de Toulon et du Var, France.

${ }^{\dagger}$ Centre de Physique Théorique, UMR 6207, CNRS, Luminy Case 907, F-13288 Marseille Cedex 9, and Universities of Aix-Marseille I, II and Toulon-Var. Fédération de Recherche des Unités de Mathématiques de Marseille. E-mail: $<$ vaienti@cpt.univ-mrs.fr>. 
distribution with error terms. This result in itself will be of interest to a much larger community than the one of dynamicists addressed in this paper.

Let $\mu$ be a probability measure on a space $\Omega$ which carries a transformation $T$, preserving $\mu$, and whose $\sigma$-algebra is generated by the joins $\mathcal{A}^{k}=\bigvee_{j=0}^{k-1} T^{-j} \mathcal{A}, k=1,2, \ldots$, of a given finite measurable partition $\mathcal{A}$. The elements of $\mathcal{A}^{k}$ are called $k$-cylinders. We assume $\mathcal{A}$ is generating, i.e. the elements of $\mathcal{A}^{\infty}$ are single points. Denote by $\chi_{A}$ the characteristic function of a (measurable) set $A$ and define the random variable:

$$
\zeta_{A}(z)=\sum_{j=1}^{\tau} \chi_{A} \circ T^{j}(z)
$$

$z \in \Omega$. The value of $\zeta_{A}$ measures the number of times a given point returns to $A$ within the time $\tau$. Typically the obsevation time $\tau$ is chosen to be $[t / \mu(A)]$ where $t$ is a parameter value. (The rescaling factor $1 / \mu(A)$ agrees with Kac's theorem.) For instance, if $\mu$ is the measure of maximal entropy on a subshift of finite type, then Pitskel [25] showed that $\zeta_{A_{n}(x)}$ is for $\mu$-almost every $x \in \Omega$ in the limit $n \rightarrow \infty$ Poisson distributed (where $\tau_{n}=\left[t / \mu\left(A_{n}(x)\right)\right]$ and $A_{n}(x)$ denotes the unique $n$-cylinder that contains $x$ ). In [13] we have proven a similar result for a much wider class of systems and provided error estimates.

We develop a mechanism which allows to prove the compound Poisson distribution of return times at periodic points $x$ and also to obtain error estimates as the cylinder sets $A_{n}(x)$ shrink in measure to zero.

To be more precise, if we denote by $\zeta_{n}^{t}(z)$ the counting function $\sum_{j=1}^{\tau_{n}} \chi_{A_{n}(x)} \circ T^{j}(z)$, with the observation time $\tau_{n}=\left[\frac{t}{(1-p) \mu\left(A_{n}(x)\right)}\right]$, we will study the following distribution:

$$
\mathbb{P}\left(\zeta_{n}^{t}=r\right), \quad r=0,1,2, \ldots,
$$

where $t>0$ is a parameter and $p \in[0,1)$ depends on the periodic point $x$ and will be given in Sect. 3 . We will show that the limit $n \rightarrow \infty$ is the compound Poisson distribution (see Section 2) if $\mu$ is a $(\phi, f)$ mixing measure. We also provide rates of convergence. This then implies under some mild additional assumptions 14 the uniform integrability of the process $\zeta_{n}^{t}$.

We then extend this result to return times, i.e. to the distribution of the process $\zeta_{n}^{t}(z)$ restricted to the cylinder $A_{n}(x)$. The measure $\mu$ is then replaced by the conditional measure $\mu_{n}=\left.\frac{1}{\mu\left(A_{n}(x)\right)} \mu\right|_{A_{n}(x)}$. We refer to this second case as the distribution of the number of visits for return times.

Our results for return times considerably improve on the work of Hirata [15, where he computed (without error) the distribution of the first return time (order $r=0$ ) around periodic points and for Gibbs measures on Axiom-A systems.

The plan of the paper is the following. The purpose of section 2 is to prove Proposition 1 that gives general conditions under which a sum of mutually dependent 0,1 -valued random variables converges to the compound Poisson distribution and provides error terms. A similar result that had been inspired by a theorem of Sevast'yanov [26], was proved and used in [13] for the Poisson distribution.

The distribution of return times is tied to the mixing properties of the invariant measure considered. For that purpose we introduce in the third section the $(\phi, f)$-mixing property. This property is more general that the widely used $\phi$-mixing property and is reminiscent of Philipp and Stout 24] 'retarded strong mixing property'. In this way one can obtain distribution results on return times of some well studied dynamical systems that are not $\phi$-mixing, e.g. rational maps, parabolic maps, piecewise expanding maps in higher dimension ....

The third section is devoted to the proof of the existence of the limit distribution and rates of convergence for entry times (Theorem 7), while the fourth section extends those results to return times (Theorem 10). Section 5 contains a careful application to rational maps with critical points (Theorem 11).

We conclude this introduction with an interesting observation. Limit distributions for entry and return times have been provided along nested sequences of cylinder sets converging to points $x$ which were chosen almost everywhere or as periodic points. In section 3.4 we will show how to find points 
$x$ which do not have limit distributions at all, and this will be achieved by using our results on the compound Poisson distribution around periodic points.

\section{Factorial moments and mixing}

The main purpose of this section is to prove a very general result which we use to prove the main results in sections 3 and 5 but which can also be useful to establish compound Poisson distribution with respect to the geometric distributiion in many other settings. For more general compound Poisson distributions see [9]. More recently (e.g. [7, 2]) there have been efforts to approach compound Poisson distributions using the Chen-Stein method. The treatment in [7] has a more general setting, but the result is far from applicable to our situation 11 Proposition 1 is of general interest and is reminiscent of existing theorems which establish the Poisson distribution (cf. [26, 13]). from the convergence of the moments. It provides general conditions under which the distribution of a finite set of 0,1 -valued random variables is close to compound Poisson (and provides error terms). In sections 3 and 5 we then use it to obtain the speed of convergence for the limiting distributions for $\phi$-mixing systems, some non-Markovian systems and equilibrium states for rational maps with critical points.

\subsection{Compound Poisson distribution}

For a parameter $p \in[0,1)$ let us define the polynomials

$$
P_{r}(t, p)=\sum_{j=1}^{r} p^{r-j}(1-p)^{j} \frac{t^{j}}{j !}\left(\begin{array}{c}
r-1 \\
j-1
\end{array}\right),
$$

$r=1,2, \ldots$, where $P_{0}=1(r=0)$. The distribution $e^{-t} P_{r}(t, p), r=0,1,2, \ldots$ is sometimes called the Pólya-Aeppli distribution [18]. It has the generating function

$$
g_{p}(z)=e^{-t} \sum_{r=0}^{\infty} z^{r} P_{r}=e^{t \frac{z-1}{1-p z}}
$$

its mean is $\frac{t}{1-p}$ and its variance is $t \frac{1+p}{(1-p)^{2}}$. Note that for $p=0$ we recover the Poisson terms $e^{-t} P_{r}(t, 0)=$ $e^{-t} \frac{t^{r}}{r !}$ and the generating function $g_{0}(z)=e^{t(z-1)}$ which is analytic in the entire plane whereas for $p>0$ the generating function $g_{p}(z)$ has an essential singularity at $\frac{1}{p}$. The expansion at $z_{0}=1$ yields $g_{p}(z)=\sum_{k=0}^{\infty}(z-1)^{k} Q_{k}$ where

$$
Q_{k}(t, p)=\frac{1}{(1-p)^{k}} \sum_{j=1}^{k} p^{k-j} \frac{t^{j}}{j !}\left(\begin{array}{c}
k-1 \\
j-1
\end{array}\right)
$$

$\left(Q_{0}=1\right)$ are the factorial moments. Note that in particular $P_{0}(0, p)=1$ and $P_{r}(0, p)=0$ for all $r \geq 1$.

\subsection{Return times patterns}

Let $M$ and $m<M$ be given integers (typically $m \ll M$ ) and let $\tau \in \mathbb{N}$ be some (large) number. For $r=1,2,3, \ldots$ we define the following:

(I) $G_{r}(\tau)$ : We denote by $G_{r}(\tau)$ the $r$-vectors $\vec{v}=\left(v_{1}, \ldots, v_{r}\right) \in \mathbb{Z}^{r}$ for which $1 \leq v_{1}<v_{2}<\cdots<v_{r} \leq \tau$. (II) $G_{r, j}(\tau)$ : We divide the set $G_{r}$ into disjoint subsets $G_{r, j}$ where $G_{r, j}$ consists of all $\vec{v} \in G_{r}$ for which we can find $j$ indices $i_{1}, i_{2}, \ldots, i_{j} \in\{1,2, \ldots, r\}, i_{1}=1$, so that $v_{k}-v_{k-1} \leq M$ if $k \neq i_{2}, \ldots, i_{j}$ and so that $v_{k}-v_{k-1}>M$ for all $k=i_{2}, \ldots, i_{j}$.

For $\vec{v} \in G_{r, j}$ the values of $v_{i}$ will be identified with returns; returns that occur within less than time $M$ are called immediate returns and if the return time is $\geq M$ then we call it a long return (i.e. if $v_{i+1}-v_{i}<M$ then we say $v_{i+1}$ is an immediate return and if $v_{i+1}-v_{i} \geq M$ the we call $v_{i}$ a long return). That means that $G_{r, j}$ consists of all return time patterns $\vec{v}$ which have $r-j$ immediate returns

\footnotetext{
${ }^{1}$ We thank the referee for pointing us towards Chen and Roos' work and also for other enlightening remarks.
} 
that are clustered into $j$ blocks of immediate returns and $j-1$ long returns between those blocks. The entries $v_{i_{k}}, k=1, \ldots, j$, are the beginnings (heads) of the blocks (of immediate returns). We assume from now on that all short returns are multiples of $m$. (This reflects the periodic structure around periodic points, cf. condition (II) of Proposition 1])

(III) $G_{r, j, w}(\tau)$ : For $\vec{v} \in G_{r, j}$ the length of each block is $v_{i_{k+1}-1}-v_{i_{k}}, k=1, \ldots, j-1$. Consequently let us put $w_{k}=\frac{1}{m}\left(v_{k}-v_{k-1}\right)$ for the individual overlaps, for $k \neq i_{1}, i_{2}, \ldots, i_{j}$. Then $\sum_{\ell=i_{k}+1}^{i_{k+1}-1} w_{\ell}=$ $\frac{1}{m}\left(v_{i_{k+1}-1}-v_{i_{k}}\right)$ is the overlap of the $k$ th block and $w=w(\vec{v})=\sum_{k \neq i_{1}, i_{2}, \ldots, i_{j}} w_{k}$ the total overlap of $\vec{v}$. We now put $G_{r, j, w}=\left\{\vec{v} \in G_{r, j}: w(\vec{v})=w\right\} .\left(G_{r, j}=\bigcup_{w} G_{r, j, w}\right.$ is a disjoint union.)

(IV) $\Delta(\vec{v})$ : For $\vec{v}$ in $G_{r, j}$ we put

$$
\Delta(\vec{v})=\min \left\{v_{i_{k}}-v_{i_{k}-1}: k=2, \ldots, j\right\}
$$

for the minimal distance between the 'tail' and the 'head' of successive blocks of immediate returns (or the length of the shortest one of the long gaps).

\subsection{Compound Poisson approximations}

The purpose of this section is to prove the following result on the approximation of the compound Poisson distribution.

Proposition 1 Let $M, m, \tau$ be as above. Let $\eta_{j}, j=1, \ldots, \tau$, be 0,1 -valued random variables on some $\Omega$ for $\vec{v} \in G_{r}$ put $\eta_{\vec{v}}=\prod_{i} \eta_{v_{i}}$. Choose $\delta>0$ and define the 'rare set' $R_{r}=\bigcup_{j=1}^{r} R_{r, j}$, where $R_{r, j}=\left\{\vec{v} \in G_{r, j}: \Delta(\vec{v})<\delta\right\}$. Let $\mu$ be a probability measure on $\Omega$ which satisfies the following conditions $\left(C_{0}\right.$ is a constant):

(I) $\mathbb{E}\left(\eta_{j}\right)=\beta$ for all $j=1, \ldots, \tau$ (invariance of the measure).

(II) Suppose that there are numbers $0<p_{-} \leq p \leq p_{+}, \phi \geq 0$ so that for all $\vec{v} \in G_{r, j, w} \backslash R_{r, j}$

$$
\left|\mathbb{E}\left(\eta_{\vec{v}}\right)-p^{w} \beta^{j}\right| \leq C_{0} \beta^{j}\left(p_{+}^{w}-p_{-}^{w}\right)+p^{w}\left((1+\phi)^{j}-1\right)
$$

if all of the individual overlaps $w_{\ell}$ are multiples of $m$, and

$$
\mathbb{E}\left(\eta_{\vec{v}}\right)=0
$$

if some of the individual overlaps $w_{\ell}$ are not multiples of $m$.

(III) There are some constants $\gamma \geq 1, \gamma_{1}, \gamma_{2}$ small $\left(\right.$ e.g. $\left.\gamma\left(\gamma_{1}+\gamma_{2}\right)<\frac{1}{12}\right)$, so that for all $r$

$$
\sum_{\vec{v} \in R_{r}} \mathbb{E}\left(\eta_{\vec{v}}\right) \leq C_{0} r \gamma^{r} \sum_{j=2}^{r} \sum_{s=1}^{j-1}\left(\begin{array}{c}
j-1 \\
s-1
\end{array}\right) \gamma_{1}^{j-s} \frac{(\tau \beta)^{s}}{s !}\left(\begin{array}{c}
r-1 \\
j-1
\end{array}\right) \gamma_{2}^{r-j}
$$

Let us put $\zeta=\sum_{j=1}^{\tau} \eta_{j}$ and $t=(1-p) \tau \beta$.

Then there exists a constant $C_{1}$ so that for every $t>0$ one has

$\left|\mathbb{P}(\zeta=r)-e^{-t} P_{r}(t, p)\right| \leq C_{1}\left(\gamma_{1}+\delta \beta\right) t^{r-1} \frac{e^{2 r}}{r !}+C_{1}\left(p_{+}^{\frac{M}{m}}+p_{+}-p_{-}+\phi\right)\left\{\begin{array}{ll}\frac{t^{r}}{r !} e^{2 r+\frac{5}{2} t} & \text { if } t>\frac{1}{2} p r \\ (2 p)^{r} e^{t \frac{1+2 p}{1-4 p}} & \text { if } \quad t \leq \frac{1}{2} p r\end{array}\right.$.

Note that the constants $\gamma$ and $\gamma_{2}$ don't enter the final estimate in an explicit way. The significant quantity here is $\gamma_{1}$ which typically is $\ll 1$ where $\gamma, \gamma_{2}$ only have to be small enough of order $\mathcal{O}(1)$.

The choice of $\delta$ is central to the application of this proposition. In the application however $\phi$ depends on $\delta$ and in fact $\phi(\delta) \rightarrow 0+$ as $\delta \rightarrow \infty$. Obviously a larger value for $\delta$ increases the error term as one sees from the expression, but also a smaller value increases the error term since the rare set $R_{r}$ becomes larger and the 'mixing property' in (II) will require larger $p_{+}$and smaller $p_{-}$, thus again increasing the error estimate. The trick is to optimise $\delta$.

Proof. We compare the generating function $\psi(z)$ for the process $\zeta$ with the generating function $g_{p}(z)$ for the compound Poissonian. In part $(\mathrm{A})$ we compare their Taylor coefficients at $z=1$ and in part 
(B) we use Cauchy estimates to compare their Taylor coefficients at $z=0$ which then gives us the final result.

(A) The coefficients at $z=1$ (factorial moments) of the generating function $\psi(z)=\sum_{r=0}^{\infty} z^{r} \mathbb{P}(\zeta=$ $r)=\sum_{r=0}^{\infty}(z-1)^{r} U_{r}$ are

$$
U_{r}=\sum_{\vec{v} \in G_{r}} \mathbb{E}\left(\eta_{\vec{v}}\right)
$$

while the coefficients of the generating function $g_{p}(z)=\sum_{r=0}^{\infty}(z-1)^{r} Q_{r}(t, p)$ for the compound Poisson distribution are

$$
Q_{r}(t, p)=\frac{1}{(1-p)^{r}} \sum_{j=1}^{r} \frac{t^{j}}{j !}\left(\begin{array}{c}
r-1 \\
j-1
\end{array}\right) p^{r-j}=\sum_{u=r-j}^{\infty} \sum_{j=1}^{r} p^{u} \beta^{j} \frac{\tau^{j}}{j !}\left(\begin{array}{c}
u-1 \\
r-j-1
\end{array}\right)\left(\begin{array}{c}
r-1 \\
j-1
\end{array}\right),
$$

where $t=(1-p) \beta \tau$. We will now compare the coefficients $Q_{r}$ to the coefficients $U_{r}$. There are three parts to the comparison: (i) Assumption (II) is used to compare the terms for which $\vec{v} \in G_{r} \backslash R_{r}$; (ii) Assumptions (I) and (III) are used to estimate the total contributions made by $\vec{v} \in R_{r}$; (iii) We have to estimate the contribution to $Q_{r}$ that correspond to overlaps $u$ which do not occur for vectors $\vec{v}$ in $G_{r}$ and therefore cannot be matched with terms in the sum that defines $U_{r}$.

More precisely, we estimate as follows:

$$
\left|U_{r}-Q_{r}((1-p) \beta \tau, p)\right| \leq \sum_{j} \sum_{u} \sum_{\vec{v} \in G_{r, j, u} \backslash R_{j}}\left|\mathbb{E}\left(\eta_{\vec{v}}\right)-p^{w} \beta^{j}\right|+\sum_{\vec{v} \in R_{r}}\left(\mathbb{E}\left(\eta_{\vec{v}}\right)+p^{u(\vec{v})} \beta^{j}\right)+V(r) .
$$

Before we proceed to bound the three terms on the right hand side let us estimate the cardinality of the sets $G_{r, j, u}$. (Note that $u \geq r-j$ if $G_{r, j, u}$ is nonempty.) Since $G_{r, j, u}$ consists of all $\vec{v} \in G_{r, j}$ that have a total overlap $u$ (in $j$ blocks of immediate returns) we get

$$
\left|G_{r, j, u}\right| \leq \frac{\tau^{j}}{j !}\left(\begin{array}{c}
u-(r-j)+r-j-1 \\
r-j-1
\end{array}\right)\left(\begin{array}{c}
r-1 \\
j-1
\end{array}\right)=\frac{\tau^{j}}{j !}\left(\begin{array}{c}
u-1 \\
r-j-1
\end{array}\right)\left(\begin{array}{c}
r-1 \\
j-1
\end{array}\right)
$$

( $j$ blocks positioned 'anywhere' on an interval of length $\tau, u$ overlaps distributed on $r-j$ immediate returns and $j$ blocks beginning on any of the $r$ return times).

Now we estimate the three terms in the coefficient comparison as follows:

(i) The first error term (difference between the dominating terms) is bounded using assumption (II):

$$
\begin{aligned}
\sum_{j=1}^{r} & \sum_{u=r-j}^{\infty} \sum_{\vec{v} \in G_{r, j, u} \backslash R_{r}}\left|\mathbb{E}\left(\eta_{\vec{v}}\right)-p^{w} \beta^{j}\right| \\
& \leq \sum_{j=1}^{r} \sum_{u=r-j}^{\infty}\left|G_{r, j, u}\right| \beta^{j}\left(p_{+}^{u}-p_{-}^{u}(1-\phi)\right) \\
& \leq \sum_{j=1}^{r} \sum_{u=r-j}^{\infty} \frac{\tau^{j}}{j !}\left(\begin{array}{c}
u-1 \\
r-j-1
\end{array}\right)\left(\begin{array}{c}
r-1 \\
j-1
\end{array}\right) \beta^{j}\left(p_{+}^{u}-p_{-}^{u}(1-\phi)\right) \\
& \leq \sum_{j=1}^{r} \frac{\tau^{j}}{j !} \beta^{j}\left(\begin{array}{c}
r-1 \\
j-1
\end{array}\right)\left(\left(\frac{p_{+}}{1-p_{+}}\right)^{r-j}-\left(\frac{p_{-}}{1-p_{-}}\right)^{r-j}(1-\phi)\right) \\
& \leq \frac{c_{1} q}{\left(1-p_{+}\right)^{2}} \sum_{j=1}^{r-1}(r-1) \frac{\tau^{j} \beta^{j}}{j !}\left(\begin{array}{c}
r-2 \\
j-1
\end{array}\right)\left(\frac{p_{+}}{1-p_{+}}\right)^{r-j-1}+\phi \sum_{j=1}^{r} \frac{\tau^{j}}{j !} \beta^{j}\left(\begin{array}{c}
r-1 \\
j-1
\end{array}\right)\left(\frac{p_{+}}{1-p_{+}}\right)^{r-j} \\
& \leq c_{2} q(r-1) Q_{r-1}\left(t, p_{+}\right)+\phi Q_{r}\left(t, p_{+}\right)
\end{aligned}
$$

(because $\left.(r-j)\left(\begin{array}{c}r-1 \\ j-1\end{array}\right)=(r-1)\left(\begin{array}{c}r-2 \\ j-1\end{array}\right)\right)$, where $t=(1-p) \tau \beta$ and $q=p_{+}-p_{-}$.

(ii) For the second term let us note that $R_{r}=\bigcup_{j} R_{r, j}$ where $R_{r, j}=\left\{\vec{v} \in G_{r, j}: \Delta(\vec{v})<\delta\right\}$. Put $R_{r}^{s}$ for those $\vec{v} \in R_{r}$ where $v_{i+1}-v_{i} \geq \delta$ for exactly $s-1$ indices $i_{1}, i_{2}, \ldots, i_{s-1}$ and put $i_{s}=v_{r}$ (obviously $1 \leq s \leq j-1 \leq r-1$ and $\left.i_{s-1} \leq r-1\right)$. 
To estimate the cardinality of $R_{r, j, u}^{s}=R_{r}^{s} \cap G_{r, j, u}$ let us note that the number of possibilities of $v_{i_{1}}<v_{i_{2}} \cdots<v_{i_{s}}$ (entrance times for long returns bigger than $\delta$ ) is bounded above by $\frac{1}{s !} \tau^{s}$ (this is the upper bound for the number of possibilities to obtain $s-1$ intervals contained in the interval $[1, \tau]$ ), and each of the remaining $j-s$ return times less than $\delta$ assume no more than $\delta$ different values. Since the indices $i_{s}, \ldots, i_{k_{s}}$ can be picked in $\left(\begin{array}{c}j-1 \\ s-1\end{array}\right)$ many ways out of $j$ blocks, we obtain:

$$
\left|R_{r, j, u}^{s}\right| \leq\left(\begin{array}{c}
j-1 \\
s-1
\end{array}\right) \frac{\delta^{j-s}}{s !} \tau^{s}\left(\begin{array}{c}
r-1 \\
j-1
\end{array}\right)\left(\begin{array}{c}
u-1 \\
r-j-1
\end{array}\right) .
$$

To estimate the contribution made by the portion of the sum in the definition of $Q_{r}$ which corresponds to the vectors $\vec{v} \in R_{r}$ we obtain by summing over $s$ :

$$
\begin{aligned}
\sum_{\vec{v} \in R_{r}} \beta^{j} p^{w(\vec{v})} & \leq \sum_{j} \sum_{s=1}^{j-1} \sum_{u=r-j}^{\infty} \beta^{j} p^{u}\left|R_{r, j, u}^{s}\right| \\
& \leq \sum_{j=2}^{r} \sum_{s=1}^{j-1}\left(\begin{array}{c}
j-1 \\
s-1
\end{array}\right) \frac{(\tau \beta)^{s}}{s !}(\delta \beta)^{j-s}\left(\begin{array}{c}
r-1 \\
j-1
\end{array}\right) \sum_{u=r-j}^{\infty}\left(\begin{array}{c}
u-1 \\
r-j-1
\end{array}\right) p^{u} \\
& \leq \sum_{j=2}^{r} \sum_{s=1}^{j-1}\left(\begin{array}{c}
j-1 \\
s-1
\end{array}\right) \frac{(\tau \beta)^{s}}{s !}(\delta \beta)^{j-s}\left(\begin{array}{c}
r-1 \\
j-1
\end{array}\right)\left(\frac{p}{1-p}\right)^{r-j} .
\end{aligned}
$$

The corresponding term for the actual expected values of $\eta_{\vec{v}}$ where $\vec{v}$ is in the rare set is bounded by assumption (II). Hence we obtain

$$
\sum_{j} \sum_{\vec{v} \in R_{r, j}}\left(\mathbb{E}\left(\eta_{\vec{v}}\right)+\beta^{j} p^{w(\vec{v})}\right) \leq S_{r}
$$

where

$$
S_{r}=c_{3} r \gamma^{r} \sum_{j=2}^{r} \sum_{s=1}^{j-1}\left(\begin{array}{c}
j-1 \\
s-1
\end{array}\right) \hat{\gamma}_{1}^{j-s} \frac{(\tau \beta)^{s}}{s !}\left(\begin{array}{c}
r-1 \\
j-1
\end{array}\right) \hat{\gamma}_{2}^{r-j},
$$

for some $c_{3}$, and $\hat{\gamma}_{1}=\gamma_{1}+\delta \beta, \hat{\gamma}_{2}=\gamma_{2}+\frac{p}{1-p}$.

(iii) Since the sum for $Q_{r}$ contains many terms that cannot be paired with terms in the sum of $U_{r}$ let us look at those combinations of $r, j$, and $u$ that do not correspond to vectors in $G_{r}$. Let us denote by $V_{r, j, u}$ the number of elements for the values of $r, j, u$ that occur in the representation of $Q_{r}$ and are not in $G_{r}$. Those overcounts occur for overlaps which have lengths $\geq M$. Denote by $\tilde{u}_{1}, \tilde{u}_{2}, \ldots, \tilde{u}_{r-j}$ the individual overlaps of these $r-j$ fictitious 'immediate returns'. Then, if the first intersection is of length $\tilde{u}_{1}\left(\geq \frac{M}{m}\right)$, then

$$
V_{r, j, u} \leq r \sum_{\tilde{u}_{1}=\frac{M}{m}}^{u-(r-j)}\left|G_{r, j, u-\tilde{u}_{1}}\right|,
$$

Hence (for $u>\frac{M}{m}+(r-j-1)$ and $j<r$ )

$$
V_{r, j, u} \leq r \frac{\tau^{j}}{j !} \sum_{\tilde{u}_{1}=\frac{M}{m}}^{u-(r-j-1)}\left(\begin{array}{c}
u-\tilde{u}_{1}-1 \\
r-j-1
\end{array}\right)\left(\begin{array}{c}
r-1 \\
j-1
\end{array}\right)=r \frac{\tau^{j}}{j !}\left(\begin{array}{c}
r-1 \\
j-1
\end{array}\right)\left(\begin{array}{c}
u-\frac{M}{m} \\
r-j-1
\end{array}\right)
$$

where we used the identity $\sum_{y=b}^{a}\left(\begin{array}{c}y-1 \\ b-1\end{array}\right)=\left(\begin{array}{c}a \\ b\end{array}\right)$. Let us put $H_{r}$ for the set of ficticious vectors $\vec{v}$ that have individual overlaps $u_{i}\left(i \neq i_{1}, \ldots i_{j}\right)$ that are not allowed, i.e. where at least one overlap is longer than $\frac{M}{m}$. Then we can estimate the contribution one gets by counting over $H_{r}$ as follows:

$$
V(r)=\sum_{\vec{v} \in H_{r}} \beta^{j} p_{+}^{u(\vec{v})}
$$




$$
\begin{aligned}
& =r \sum_{j=1}^{r-1} \sum_{u=\frac{M}{m}+r-j}^{\infty} V_{r, j, u} \beta^{j} p_{+}^{u} \\
& \leq r \sum_{j=1}^{r-1} \beta^{j} \frac{\tau^{j}}{j !}\left(\begin{array}{c}
r-1 \\
j-1
\end{array}\right) \sum_{u=\frac{M}{m}+r-j-1}^{\infty} p_{+}^{u}\left(\begin{array}{c}
u-\frac{M}{m} \\
r-j-1
\end{array}\right) \\
& =r \sum_{j=1}^{r-1} \beta^{j} \frac{\tau^{j}}{j !}\left(\begin{array}{c}
r-1 \\
j-1
\end{array}\right) p_{+}^{\frac{M}{m}-1}\left(\frac{p_{+}}{1-p_{+}}\right)^{r-j}
\end{aligned}
$$

where we used the identity $\sum_{u=a}^{\infty} p^{u}\left(\begin{array}{c}u-1 \\ a-1\end{array}\right)=\left(\frac{p}{1-p}\right)^{a}$. Hence

$$
V(r) \leq r p_{+}^{\frac{M}{m}-1} \sum_{j=1}^{r-1} \beta^{j} \frac{\tau^{j}}{j !}\left(\begin{array}{c}
r-1 \\
j-1
\end{array}\right)\left(\frac{p_{+}}{1-p_{+}}\right)^{r-j}=r p_{+}^{\frac{M}{m}-1} Q_{r}\left(t, p_{+}\right) .
$$

Combining the three estimates (i), (ii) and (iii) yields

$$
\left|U_{r}-Q_{r}(t, p)\right| \leq\left(r p^{\frac{M}{m}-1}+\phi\right) Q_{r}\left(t, p_{+}\right)+c_{5} q(r-1) Q_{r-1}\left(t, p_{+}\right)+S_{r}
$$

(B) The difference $\varphi(z)=\psi(z)-g_{p}(z)$ between the two generating functions splits into two parts, $\varphi=\varphi_{1}+\varphi_{2}$, which we analyse separately $\left(\varphi_{1}\right.$ reflects the estimates of parts (A-i) and (A-iii), and $\varphi_{2}$ reflects the estimate of (A-ii)):

(i) The function $\varphi_{1}(z)$ is majorised by the power series

$$
c_{6}\left(p^{\frac{M}{m}}+q+\phi\right) \sum_{r}|z-1|^{r} r Q_{r}\left(t, p_{+}\right) .
$$

The sum over $r$ is equal to $\frac{d}{d w} e^{t \frac{w}{1-p_{+}-p_{+} w}}$ (where $\left.w=|z-1|\right)$ which can be bounded by $4 e^{t \frac{w}{1-p_{+}-p_{+} w}}$ if $|w| \leq \frac{3}{4} \frac{1-p_{+}}{p_{+}}$. A Cauchy estimate with $|z|=R$ now yields

$$
E_{1}=\frac{1}{r !}\left|\varphi_{1}^{(r)}(0)\right| \leq \frac{4}{R^{r}} e^{t \frac{R+1}{1-2 p_{+}-p_{+} R}}
$$

If $t$ is large so that $\frac{r}{t}<\frac{1}{2 p}$ then we can take $R=\frac{r}{t}$ and obtain for instance that (assuming $p_{+}<\frac{1}{20}$ )

$$
E_{1} \leq c_{7} \epsilon\left(\frac{t}{r}\right)^{r} e^{\frac{5}{2}(r+t)} \leq c_{8} \epsilon \frac{t^{r}}{r !} e^{2 r+\frac{5}{2} t}
$$

using Stirling's formula, where $\epsilon=c_{6}\left(p_{+}^{\frac{M}{m}}+q+\phi\right)$. If $t$ is small so that for instance $\frac{r}{t} \geq \frac{1}{2 p}$ then we take $R=\frac{1}{2 p}$ and thus obtain $E_{1}=\frac{1}{r !}\left|\varphi_{1}^{(r)}(0)\right| \leq c_{8} \epsilon(2 p)^{r} e^{t \frac{1+2 p}{1-4 p}}$.

(ii) The second error function $\varphi_{2}(z)$ is majorised by the power series $\left(t^{\prime}=\beta \tau\right)$

$$
\begin{aligned}
\sum_{r}|z-1|^{r} S_{r} & =\sum_{r=2}^{\infty}|z-1|^{r} \gamma^{r} \sum_{j=2}^{r} \sum_{s=1}^{j-1}\left(\begin{array}{c}
j-1 \\
s-1
\end{array}\right) \frac{t^{\prime s}}{s !}\left(\begin{array}{c}
r-1 \\
j-1
\end{array}\right) \hat{\gamma}_{1}^{j-s} \hat{\gamma}_{2}^{r-j} \\
& =\exp \frac{t^{\prime} \gamma|z-1|}{1-\left(\hat{\gamma}_{1}+\hat{\gamma}_{2}\right) \gamma|z-1|}-\exp \frac{t^{\prime} \gamma|z-1|}{1-\hat{\gamma}_{2} \gamma|z-1|} \\
& \leq 6 \gamma \hat{\gamma}_{1}|z-1| \exp \frac{t^{\prime} \gamma|z-1|}{1-\left(\hat{\gamma}_{1}+\hat{\gamma}_{2}\right) \gamma|z-1|}
\end{aligned}
$$

if $|z-1| \gamma\left(\hat{\gamma}_{1}+\hat{\gamma}_{2}\right)$ is small enough (e.g. $\left.\leq \frac{1}{3}\right)$, where we have used the identity

$$
\sum_{r=2}^{\infty} \sum_{j=2}^{r} \sum_{s=1}^{j-1}\left(\begin{array}{c}
j-1 \\
s-1
\end{array}\right) \frac{x^{s}}{s !}\left(\begin{array}{c}
r-1 \\
j-1
\end{array}\right) y^{j-s} z^{r-j}=\exp \frac{x}{1-y-z}-\exp \frac{x}{1-z}
$$


(develop into a Taylor series with variable $x$ and use the identity $\sum_{k=\ell}^{\infty}\left(\begin{array}{c}k-1 \\ \ell-1\end{array}\right) b^{k-\ell}=(1-b)^{-\ell}$ ). Hence if we put $|z|=R(R>1)$ then

$$
E_{2}=\frac{1}{r !}\left|\varphi_{2}^{(r)}(0)\right| \leq c_{9} \gamma \hat{\gamma}_{1} \frac{e^{2 \gamma R t^{\prime}}}{R^{r-1}}
$$

if we assume that $(R+1) \gamma \hat{\gamma}_{1}$ is small enough (e.g. $<\frac{1}{3}$ ). If $R=\frac{r}{2 t^{\prime}}$ then we get (using Stirling's formula)

$$
\frac{1}{r !}\left|\varphi_{2}^{(r)}(0)\right| \leq c_{10} \hat{\gamma}_{1} 2^{r} r^{2} t^{r-1} \frac{e^{r}}{r !}
$$

(if $t^{\prime}$ is close enough to $t$ ).

Since $\mathbb{P}(\zeta=r)=\frac{1}{r !} \psi^{(r)}(0)$ and $e^{-t} P_{r}(t, p)=g_{p}^{(r)}(0)$ we get by combining the estimates (i) and (ii)

$$
\left|\mathbb{P}(\zeta=r)-e^{-t} P_{r}(t, p)\right| \leq \frac{1}{r !}\left|\varphi^{(r)}(0)\right| \leq E_{1}+E_{2}
$$

(and $1+\log 2<2$ ) from which follows the result of the proposition.

In the following we will apply this proposition to situations that typically arise in dynamical systems. There the stationarity condition (I) of the proposition is implied by the invariance of the measure. The random variables $\eta_{j}$ will be the indicator function of a cylinder set pulled back under the $j$ th iterate of the map. Condition (II) is then implied by the mixing property (see below Definition 2). The most difficult condition to satisfy is (III) because it involves 'short range' interaction over which one has little control and which require more delicate estimates (see Lemma 5 below). A simpler version of Proposition 1 is the following corollary $(m=1)$ which is easily deduced by putting $\gamma_{2}=0, \gamma=1, \gamma_{1}=\varepsilon$.

Corollary 2 Let $M, \tau$ be as above. Let $\eta_{j}, j=1, \ldots, \tau$, be 0,1 -valued random variables and $\eta_{\vec{v}}=\prod_{i} \eta_{v_{i}}$ for $\vec{v} \in G_{r}$. For some $\delta>0$ let $R_{r}=\bigcup_{j=1}^{r} R_{r, j}$, where $R_{r, j}=\left\{\vec{v} \in G_{r, j}: \Delta(\vec{v})<\delta\right\}$. Assume $\mu$ be a probability measure on $\Omega$ which satisfies the following conditions $\left(C_{0}\right.$ is a constant, $\left.\varepsilon>0\right)$ :

(I) $\mathbb{E}\left(\eta_{j}\right)=\beta$ for all $j=1, \ldots, \tau$ (invariance of the measure);

(II) Suppose there is a $p \in(0,1)$ so that for all $\vec{v} \in G_{r, j, w} \backslash R_{r, j}$ :

$$
\left|\mathbb{E}\left(\eta_{\vec{v}}\right)-p^{w} \beta^{j}\right| \leq \varepsilon p^{w} ;
$$

for all $r, j$ and $w$;

(III)

$$
\sum_{\vec{v} \in R_{r}} \mathbb{E}\left(\eta_{\vec{v}}\right) \leq \varepsilon
$$

for all $r=1,2, \ldots$.

Then there exists a constant $C_{1}$ so that for every $t>0$ one has $\left(\zeta=\sum_{j=1}^{\tau} \eta_{j}\right.$ and $\left.t=(1-p) \tau \beta\right)$

$$
\left|\mathbb{P}(\zeta=r)-e^{-t} P_{r}(t, p)\right| \leq C_{1}(\varepsilon+\delta \beta) t^{r-1} \frac{e^{2 r}}{r !}+C_{1}\left(p^{M}+\varepsilon\right)\left\{\begin{array}{ll}
\frac{t^{r}}{r !} e^{2 r+\frac{5}{2} t} & \text { if } t>\frac{1}{2} p r \\
(2 p)^{r} e^{t \frac{1+2 p}{1-4 p}} & \text { if } t \leq \frac{1}{2} p r
\end{array} .\right.
$$

\section{Measures that are $(\phi, f)$-mixing}

Let $T$ be a map on a space $\Omega$ and $\mu$ a probability measure on $\Omega$. Moreover let $\mathcal{A}$ be a measurable partition of $\Omega$ and denote by $\mathcal{A}^{n}=\bigvee_{j=0}^{n-1} T^{-j} \mathcal{A}$ its $n$-th join which also is a measurable partition of $\Omega$ for every $n \geq 1$. The atoms of $\mathcal{A}^{n}$ are called $n$-cylinders. Let us put $\mathcal{A}^{*}=\bigcup_{n=1}^{\infty} \mathcal{A}^{n}$ for the collection of all cylinders in $\Omega$ and put $|A|$ for the length of an $n$-cylinder $A \in \mathcal{A}^{*}$, i.e. $|A|=n$ if $A \in \mathcal{A}^{n}$.

We shall assume that $\mathcal{A}$ is generating, i.e. that the atoms of $\mathcal{A}^{\infty}$ are single points in $\Omega$.

In the following definition we generalise the 'retarded strong mixing condition' (see e.g. 24). We consider mixing dynamical systems in which the function $\phi$ determines the rate of mixing while the separation function $f$ specifies a lower bound for the size of the gap $m$ that is necessary to get the mixing property. 
Definition 3 Assume $\mu$ is a T-invariant probability measure on $\Omega$ and that there are functions $f$ and $\phi$ so that:

(i) $f: \mathbb{N} \rightarrow \mathbb{N}_{0}\left(\mathbb{N}_{0}=\mathbb{N} \cup\{0\}\right)$ is non-decreasing

(ii) $\phi: \mathbb{N}_{0} \rightarrow \mathbb{R}^{+}$is non-increasing.

We say that the dynamical system $(T, \mu)$ is $(\phi, f)$-mixing if

$$
\left|\mu\left(U \cap T^{-m-n} V\right)-\mu(U) \mu(V)\right| \leq \phi(m) \mu(U) \mu(V)
$$

for all $m \geq f(n), n \geq 0$, measurable $V$ (in the $\sigma$-algebra generated by $\mathcal{A}^{*}$ ) and $U$ which are unions of $n$-cylinders.

Systems that are $(\phi, f)$-mixing are for instance:

1. Classical $\phi$-mixing systems (see, e.g. [6]): $f=0$. These include equilibrium states for Hölder continuous potentials on Axiom A systems (which include subshifts of finite type) or on the Julia set of hyperbolic rational maps. In this case the partition $\mathcal{A}$ is finite.

2. Dispersing billiards [23]: $f$ is linear.

3. Equilibrium states for Hölder continuous potentials (that satisfy the supremum gap (see section 5) on the Julia set of rational maps where the Julia set contains critical points: $f$ is linear, $\phi$ is exponential.

4. Multidimensional piecewise continuous maps 22]: $f$ depends on the individual cylinders $(|\mathcal{A}|<$ $\infty)$.

For $r \geq 1$ and (large) $\tau \in \mathbb{N}$ let as above $G_{r}(\tau)$ be the $r$-vectors $\vec{v}=\left(v_{1}, \ldots, v_{r}\right) \in \mathbb{Z}^{r}$ for which $1 \leq v_{1}<v_{2}<\cdots<v_{r} \leq \tau$. Let $t$ be a positive parameter, $W \subset \Omega$ and put $\tau=[t / \mu(W)]$ be the normalised time. Then the entries $v_{j}$ of the vector $\vec{v} \in G_{r}(\tau)$ are the times at which all the points in $C_{\vec{v}}=\bigcap_{j=1}^{r} T^{-v_{j}} W$ hit the set $W$ during the time interval $[1, \tau]$.

Lemma 4 Let $(T, \mu)$ be $(\phi, f)$-mixing.

Then for all $r>1, W_{i} \subset \Omega$ unions of $n_{i}$-cylinders, $i=1, \ldots, r\left(n_{i} \geq 1\right)$, and all 'hitting vectors' $\vec{v} \in G_{r}(\tau)$ with return times $v_{i+1}-v_{i} \geq f\left(n_{i}\right)+n_{i}(i=1, \ldots, r-1)$ one has

$$
\left|\frac{\mu\left(\bigcap_{i=1}^{r} T^{-v_{i}} W_{i}\right)}{\prod_{i=1}^{r} \mu\left(W_{i}\right)}-1\right| \leq(1+\phi(d(\vec{v}, \vec{n})))^{r}-1,
$$

and $d(\vec{v}, \vec{n})=\min _{i}\left(v_{i+1}-v_{i}-n_{i}\right)$.

A consequence of this is that there exists a $0<\eta<1$ so that for all $\mu(A) \leq \eta^{|A|}$ for all $A \in \mathcal{A}^{*}$.

\subsection{Estimate of the rare set}

In this section we provide an estimate for the rare set for general $(\phi, f)$-mixing maps. We will then use this result in its full strength later to show that the return times distribution at periodic points is compound Poissonian for rational maps that have critical points. For a 'hitting vector' $\vec{v} \in G_{r}(\tau)(\tau$ a large integer) we put $C_{\vec{v}}=\bigcap_{k=1}^{r} T^{-v_{k}} W$. Let $\delta \geq f(|W|)$ (W a union of cylinders of the same lengths) then

$$
R_{r, j}(\tau)=\left\{\vec{v} \in G_{r, j}(\tau): \min _{k}\left(v_{i_{k}+1}-v_{i_{k}}-|W|\right)<\delta\right\}
$$

where the values $v_{i_{1}}, \ldots, v_{i_{j}}$ are the beginnings of the $j$ blocks of immediate returns (notation as in section $2.2(\mathrm{II}))$.

Lemma 5 Assume $(T, \mu)$ is $(\phi, f)$-mixing and assume that there is an $m \in \mathbb{N}$ so that for every $n$ for $\{$ R.small $\}$ which $f(n) \leq \delta$ there exists an $M<n$ so that $A_{n} \cap T^{-\ell} A_{n} \neq \emptyset$ for $\ell<M$ implies that $\ell$ is a multiple of $m$. 
Then there exists a constant $C_{2}$ so that for all n-cylinders $A_{n}$ :

$$
\sum_{\vec{v} \in R_{r}} \mu\left(C_{\vec{v}}\right) \leq C_{2} \gamma^{r-1} \sum_{j=2}^{r} \sum_{s=1}^{j-1}\left(\begin{array}{c}
j-1 \\
s-1
\end{array}\right)\left(\delta \mu\left(A_{n^{\prime}}\right)\right)^{j-s} \frac{\left(\tau \mu\left(A_{n}\right)\right)^{s}}{s !}\left(\begin{array}{c}
r-1 \\
j-1
\end{array}\right)\left(\gamma \mu\left(A_{m^{\prime}}\right)\right)^{r-j},
$$

where:

(i) $n^{\prime}, m^{\prime}\left(m^{\prime} \leq n^{\prime}\right)$ satisfy $f\left(n^{\prime}\right) \leq M-n^{\prime}$ and $f\left(m^{\prime}\right) \leq m-m^{\prime}$,

(ii) $\gamma>1+\phi\left(\min _{i}\left(v_{i+1}-v_{i}\right)-n^{\prime}\right)$,

(iii) $A_{n^{\prime}} \in \mathcal{A}^{n^{\prime}}, A_{n} \subset A_{n^{\prime}}$,

(iv) $A_{m^{\prime}} \in \mathcal{A}^{m^{\prime}}, A_{n^{\prime}} \subset A_{m^{\prime}}$.

Proof. As in section (A-ii) of the proof of Proposition 1, put $R_{r, j}^{s}$ for those $\vec{v} \in R_{r, j}$ for which $v_{i+1}-v_{i} \geq \delta$ for $s-1$ indices $i_{1}, \ldots, i_{s-1}\left(i_{s}=v_{r}, s \leq j-1\right)$. We consider two separate cases: (I) $s \geq 2$ and (II) $s=1$.

(I) Assume $s \geq 2$ and $i_{1}, i_{2}, \ldots, i_{s-1}$ be the indices for which $v_{i_{k}+1}-v_{i_{k}} \geq \delta \geq f(n)$ for $k=1, \ldots, s-1$. All the other differences are $\geq M$ and smaller than $\delta$. Let $A_{n^{\prime}}$ be an $n^{\prime}$-cylinder so that $A_{n} \subset A_{n^{\prime}}$ where $n^{\prime}$ is so that $f\left(n^{\prime}\right) \leq M-n^{\prime}$. Let $j$ be the number of blocks (i.e. $\vec{v} \in G_{r, j}$ ) and let $i_{1}^{\prime}, \ldots, i_{j}^{\prime}$ be the beginnings of the 'blocks of immediate returns' (clearly $s \leq j-1$ ). There are $r-j$ immediate short returns of lengths $\in[m, n)$. Let us put

$$
\begin{aligned}
W_{i_{k}} & =A_{n} \quad \text { for } k=1, \ldots, s, \\
W_{i_{k}^{\prime}} & =A_{n^{\prime}} \quad \text { for } \quad k=1, \ldots, j, \\
W_{i} & =A_{m^{\prime}} \cap T^{-m} A_{m^{\prime}} \cap T^{-2 m} A_{m^{\prime}} \cap \cdots \cap T^{-\left(u_{i}-1\right) m} A_{m^{\prime}} \quad \text { for all } i \notin\left\{i_{k}: k\right\} \cup\left\{i_{k}^{\prime}: k\right\}
\end{aligned}
$$

where $u_{i}$ is the overlap for the $i$ th return (which is an immediate periodic return). By our choice of $n^{\prime}$ we have achieved that $v_{i_{k}+1}-v_{i_{k}} \geq \delta \geq f(n)$ and $v_{i+1}-v_{i} \geq f\left(n^{\prime}\right)$ for $i \neq i_{k}, k=1, \ldots, s-1$ and $i \neq i_{k}^{\prime}, k=1, \ldots, j$. By Lemma 4 we obtain

$$
\mu\left(C_{\vec{v}}\right) \leq \mu\left(\bigcap_{i=1}^{r} T^{-v_{i}} W_{i}\right) \leq \alpha_{1}^{r-1} \prod_{i=1}^{r} \mu\left(W_{i}\right) \leq \alpha_{1}^{r-1} \mu\left(A_{n^{\prime}}\right)^{j-s} \mu\left(A_{n}\right)^{s}\left(\alpha_{2} \mu\left(A_{m^{\prime}}\right)\right)^{u},
$$

$\left(\alpha_{1}=1+\min \left(\phi(\delta-n), \phi\left(n-n^{\prime}\right)\right), \alpha_{2}=1+\phi\left(m-m^{\prime}\right)\right)$ where the components of $\vec{n}=\left(n_{1}, \ldots, n_{r}\right)$ are given by $n_{i_{k}}=n$ for $k=1, \ldots, s$ and $n_{i}=n^{\prime}$ for $\left.i \neq i_{k}, k=1, \ldots, s\right)$, where $u=\sum_{i} u_{i}$ is the total overlap.

The cardinality of $R_{r, j, u}^{s}=R_{r}^{s} \cap G_{r, j, u}$ has been estimated in part (A-ii) of Proposition 1 to be

$$
\left|R_{r, j, u}^{s}\right| \leq\left(\begin{array}{c}
j-1 \\
s-1
\end{array}\right) \frac{\delta^{j-s}}{s !} \tau^{s}\left(\begin{array}{c}
r-1 \\
j-1
\end{array}\right)\left(\begin{array}{c}
u-1 \\
r-j-1
\end{array}\right)
$$

Therefore

$$
\sum_{\vec{v} \in R_{r, j, u}^{s}} \mu\left(C_{\vec{v}}\right) \leq \alpha_{1}^{r-1}\left(\begin{array}{c}
j-1 \\
s-1
\end{array}\right) \frac{\left(\tau \mu\left(A_{n}\right)\right)^{s}}{s !}\left(\delta \mu\left(A_{n^{\prime}}\right)\right)^{j-s}\left(\begin{array}{c}
r-1 \\
j-1
\end{array}\right)\left(\begin{array}{c}
u-1 \\
r-j-1
\end{array}\right)\left(\alpha_{2} \mu\left(A_{m^{\prime}}\right)\right)^{u} .
$$

(II) If $s=1$ then all returns between blocks are less than $\delta$ for all $k$. In the same way as above we obtain

$$
\sum_{\vec{v} \in R_{r, j, u}^{1}} \mu\left(C_{\vec{v}}\right) \leq \alpha_{1}^{r-1} \tau \mu\left(A_{n}\right)\left(\delta \mu\left(A_{n^{\prime}}\right)\right)^{j-1}\left(\begin{array}{c}
r-1 \\
j-1
\end{array}\right)\left(\begin{array}{c}
u-1 \\
r-j-1
\end{array}\right)\left(\alpha_{2} \mu\left(A_{m^{\prime}}\right)\right)^{u}
$$

Summing over $s$ and using the estimates from (I) and (II) yields

$$
\sum_{\vec{v} \in R_{r}} \mu\left(C_{\vec{v}}\right)=\sum_{j} \sum_{s=1}^{j-1} \sum_{u=r-j}^{\infty} \sum_{\vec{v} \in R_{r, j, u}^{s}} \mu\left(C_{\vec{v}}\right)
$$




$$
\begin{aligned}
& \leq \sum_{j=2}^{r} \alpha_{1}^{r-1} \sum_{s=1}^{j-1}\left(\begin{array}{c}
j-1 \\
s-1
\end{array}\right) \frac{\left(\tau \mu\left(A_{n}\right)\right)^{s}}{s !}\left(\delta \mu\left(A_{n^{\prime}}\right)\right)^{j-s}\left(\begin{array}{c}
r-1 \\
j-1
\end{array}\right) \sum_{u=r-j}^{\infty}\left(\begin{array}{c}
u-1 \\
r-j-1
\end{array}\right)\left(\alpha_{2} \mu\left(A_{m^{\prime}}\right)\right)^{u} \\
& \leq \sum_{j=2}^{r} \alpha_{1}^{r-1} \sum_{s=1}^{j-1}\left(\begin{array}{c}
j-1 \\
s-1
\end{array}\right) \frac{\left(\tau \mu\left(A_{n}\right)\right)^{s}}{s !}\left(\delta \mu\left(A_{n^{\prime}}\right)\right)^{j-s}\left(\begin{array}{c}
r-1 \\
j-1
\end{array}\right)\left(\frac{\alpha_{2} \mu\left(A_{m^{\prime}}\right)}{1-\alpha_{2} \mu\left(A_{m^{\prime}}\right)}\right)^{r-j}
\end{aligned}
$$

The lemma now follows since $\frac{\alpha_{2} \mu\left(A_{m^{\prime}}\right)}{1-\alpha_{2} \mu\left(A_{m^{\prime}}\right)} \leq \alpha^{\prime} \mu\left(A_{m^{\prime}}\right)$ with a $\alpha^{\prime}$ which is slightly larger than $\alpha_{2}$. Now we write $\alpha_{2}$ instead of $\alpha^{\prime}$.

In the case of classical $\phi$-mixing maps (see subsection 3.2 below), when $f$ is zero, we get the following simpler result. (We simply put $n^{\prime}=n$ and $m^{\prime}=m$ which then results in $A_{n^{\prime}}=A_{n}$ and $A_{m^{\prime}}=A_{m}$.)

Corollary 6 Assume $(T, \mu)$ is $\phi$-mixing and assume that there is an $m \in \mathbb{N}$ so that for every $n$ there $\{$ R.small.phi-mix exists an $M<n$ so that $A_{n} \cap T^{-\ell} A_{n} \neq \emptyset$ for $\ell<M$ implies that $\ell$ is a multiple of $m$.

Then there exists a constant $C_{3}$ so that for all $n$-cylinders $A_{n}$ :

$$
\sum_{\vec{v} \in R_{r}} \mu\left(C_{\vec{v}}\right) \leq C_{3} \alpha^{r-1} \sum_{j=2}^{r} \sum_{s=1}^{j-1}\left(\begin{array}{c}
j-1 \\
s-1
\end{array}\right)\left(\delta \mu\left(A_{n}\right)\right)^{j-s} \frac{\left(\tau \mu\left(A_{n}\right)\right)^{s}}{s !}\left(\begin{array}{c}
r-1 \\
j-1
\end{array}\right)\left(\alpha \mu\left(A_{m}\right)\right)^{r-j},
$$

where $\alpha=1+\phi(0)$ and $A_{m} \in \mathcal{A}^{m}$ contains $A_{n}$.

\section{$3.2 \phi$-mixing measures}

We say that the dynamical system $(T, \mu)$ is $\phi$-mixing if $f$ is identically zero, i.e.

$$
\left|\mu\left(U \cap T^{-m-n} V\right)-\mu(U) \mu(V)\right| \leq \phi(m) \mu(U) \mu(V)
$$

for all $m$, measurable $V$ (in the $\sigma$-algebra generated by $\mathcal{A}^{*}$ ) and $U$ which are unions of cylinders of the same length $n$, for all $n$. The function $\phi$ is assumed to be monotonically decreasing to zero.

Let $W$ be a set in $\Omega$. Then the entries $v_{i}$ of the vector $\vec{v} \in G_{r}(\tau)$ are the times at which all the points in $C_{\vec{v}}=\bigcap_{i=1}^{r} T^{-v_{i}} W$ hit the set $W$ during the time interval $[1, \tau]$. Following Lemma 4 we get that for $n_{i}$-cylinders $W_{i} \subset \Omega, i=1, \ldots, r$ :

$$
\left|\frac{\mu\left(\bigcap_{i=1}^{r} T^{-v_{i}} W_{i}\right)}{\prod_{i=1}^{r} \mu\left(W_{i}\right)}-1\right| \leq(1+\phi(d(\vec{v})))^{r}-1,
$$

for all 'hitting vectors' $\vec{v} \in G_{r}(\tau)$ with return times $v_{i+1}-v_{i} \geq n_{i}(i=1, \ldots r-1)$ where $d(\vec{v})=$ $\min _{i}\left(v_{i+1}-v_{i}-n_{i}\right)$.

\subsection{Distribution near periodic points for $\phi$-mixing measures}

Lemma 7 Let $x$ be a periodic point with minimal period $m$. If $\mu$ is $\phi$-mixing then the limit

$$
p=\lim _{\ell \rightarrow \infty}\left|\frac{1}{\ell} \log \mu\left(A_{\ell m}(x)\right)\right|
$$

exists.

Proof. We show that the quantity inside the logarithms is nearly superadditive. Let $\Delta$ be an integer so that $\phi(\Delta m) \leq \frac{1}{2}$. Then we have

$$
\left|\log \mu\left(A_{k m+\Delta m+\ell m}(x)\right)\right| \geq\left|\log \mu\left(A_{k m} \cap T^{-k m-\Delta m} A_{\ell m}\left(T^{-k m-\Delta m} x\right)\right)\right|
$$

and by the mixing property

$$
\mu\left(A_{k m} \cap T^{-k m-\Delta m} A_{\ell m}(x)\right)=\mu\left(A_{k m}\right) \mu\left(A_{\ell m}(x)\right)\left(1+\mathcal{O}^{*}(\phi(\Delta m))\right)
$$


(where $\mathcal{O}^{*}$ means that $\left|\frac{\mathcal{O}^{*}(\varepsilon)}{\varepsilon}\right| \leq 1$ for all $\varepsilon$ ). If we put $a_{j}=\left|\log \mu\left(A_{j m}(x)\right)\right|$, then

$$
a_{k+\Delta+\ell} \geq a_{k}+a_{\ell}-|\log (1-\phi(\Delta m))| \geq a_{k}+a_{\ell}-2 \phi(\Delta m)
$$

for all positive integers $k, \ell$. Iterating this inequality yields

$$
\begin{aligned}
\frac{a_{r k+(r-1) \Delta+s}}{(r k+(r-1) \Delta+s) m} & \geq \frac{r a_{k}-2(r-1) \phi(\Delta m)}{(r k+(r-1) \Delta+s) m} \\
& \geq \frac{1}{1+\frac{\Delta}{k}+\frac{s}{k r}} \frac{a_{k}}{k m}-\frac{2 \phi(\Delta m)}{k+\Delta}
\end{aligned}
$$

for positive integers $k, r$ and $s \in[0, k+\Delta)$. If we put $n=k r+(r-1) \Delta+s, 0 \leq s \leq k+\Delta-1$, and let $r \rightarrow \infty$ we obtain

$$
\liminf _{n \rightarrow \infty} \frac{a_{n}}{n m} \geq \frac{1}{1+\frac{\Delta}{k}} \frac{a_{k}}{k m}-\frac{2 \phi(\Delta m)}{k+\Delta} .
$$

Now let $k \rightarrow \infty$ and we finally get

$$
\liminf _{n \rightarrow \infty} \frac{a_{n}}{n m} \geq \limsup _{k \rightarrow \infty} \frac{a_{k}}{k m}
$$

which implies the lemma.

As a consequence of the lemma we see that $p \leq \eta^{m}$ for some $\eta<1$. (This follows from the fact that $m$-cylinders have measure $\leq \eta^{m}$ for some $\eta<1$ [13.) In particular $p$ is always strictly less than 1 .

In the following we shall assume the stronger property that $p=\lim _{n \rightarrow \infty} \frac{\mu\left(A_{n+m}(x)\right)}{\mu\left(A_{n}(x)\right)}$. This of course implies the limit in the lemma, but we are not sure whether the reverse implication is generally true. Also put $q_{n}=\sup _{\ell \geq n}\left|\frac{\mu\left(A_{\ell+m}(x)\right)}{\mu\left(A_{\ell}(x)\right)}-p\right|$. For $t>0$ and integers $n$ we put $\zeta_{n}^{t}$ for the counting function $\sum_{j=0}^{\tau_{n}} \chi_{\left.A_{n}(x)\right)} \circ T^{j}$ with the observation time

$$
\tau_{n}=\left[\frac{t}{(1-p) \mu\left(A_{n}(x)\right)}\right]
$$

(where $x$ is periodic with minimal period $m$ ).

In order to satisfy the assumptions of Proposition 1 we put $\gamma=\alpha, \gamma_{1}=\alpha \delta_{n} \mu\left(A_{n}\right)$ and $\left.\gamma_{2}=\alpha \mu\left(A_{m}\right)\right)$ and Corollary 6 .

Theorem 8 Let $(\mu, \Omega)$ be a $\phi$-mixing measure with partition $\mathcal{A}$ (finite or infinite), $x$ a periodic point $\{$ phi-mixing $\}$ with minimal period $m$ and $p$ and $q_{n}$ as above.

Then there exists a constant $C_{4}$ so that for every $\delta>0$ and every $t>0$ one has

$$
\left|\mathbb{P}\left(\zeta_{n}^{t}=r\right)-e^{-t} P_{r}\right| \leq C_{4} \delta \mu\left(A_{n}\right) t^{r-1} \frac{e^{2 r}}{r !}+C_{4}\left(p^{\frac{n}{m}}+q_{n}+\phi(\delta)\right) \begin{cases}\frac{t^{r}}{r !} e^{2 r+\frac{5}{2} t} & \text { if } t>\frac{1}{2} p r \\ (2 p)^{r} e^{t \frac{1+2 p}{1-4 p}} & \text { if } \quad t \leq \frac{1}{2} p r\end{cases}
$$

Proof. We use Proposition 1 and have to verify conditions (I)-(III). From the definition of $p$ and $q_{n}$ assumption (I) is clearly satisfied with $p_{ \pm}=p \pm q_{n}$.

To verify condition (II) let $\vec{v} \in G_{r, j, u}$ and let us look at the measure of $\mu\left(C_{\vec{v}}\right)$. By (2) we have $(\Delta$ is as defined in section 2.2 )

$$
\left|\mu\left(C_{\vec{v}}\right)-\prod_{k=1}^{j} \mu\left(D_{k}\right)\right| \leq\left((1+\phi(\Delta(\vec{v})-n))^{j}-1\right) \prod_{k=1}^{j} \mu\left(D_{k}\right),
$$

where $D_{k}$ is the $k$ th block, i.e.

$$
D_{k}=\bigcap_{\ell=i_{k}}^{i_{k+1}-1} T^{-v_{\ell}} A_{n}(x)
$$


Since $\mu\left(D_{k}\right)=\mu\left(A_{n+m u_{k}}\left(T^{v_{i_{k}}}(x)\right)\right)$ we get by definition of $q_{n}$

$$
\begin{aligned}
\frac{\mu\left(D_{k}\right)}{\mu\left(A_{n}\right)} & =\frac{\mu\left(A_{n+m u_{k}}\right)}{\mu\left(A_{n}\right)} \\
& =\frac{\mu\left(A_{n+m}\right)}{\mu\left(A_{n}\right)} \frac{\mu\left(A_{n+2 m}\right)}{\mu\left(A_{n+m}\right)} \cdots \frac{\mu\left(A_{n+m u_{k}}\right)}{\mu\left(A_{n+m\left(u_{k}-1\right)}\right)} \\
& =\left(p+\mathcal{O}\left(q_{n}\right)\right)^{u_{k}}
\end{aligned}
$$

and therefore

$$
\prod_{k=1}^{j} \frac{\mu\left(D_{k}\right)}{\mu\left(A_{n}\right)}=\prod_{k=1}^{j}\left(p+\mathcal{O}\left(q_{n}\right)\right)^{u_{k}}=\left(p+\mathcal{O}\left(q_{n}\right)\right)^{u},
$$

where $u=\sum_{k=1}^{j} u_{k}$. Hence

$$
\left|\prod_{k=1}^{j} \mu\left(D_{k}\right)-p^{u} \mu\left(A_{n}\right)^{j}\right| \leq \mu\left(A_{n}\right)^{j}\left(\left(p+q_{n}\right)^{u}-p^{u}\right)
$$

and consequently

$$
\left|\mu\left(C_{\vec{v}}\right)-p^{u} \mu\left(A_{n}\right)^{j}\right| \leq \mu\left(A_{n}\right)^{j}\left(\left(p+q_{n}\right)^{u}-p^{u}+p^{u}\left((1+\phi(\Delta(\vec{v})-n))^{j}-1\right)\right) .
$$

Hence, if $\vec{v} \notin R_{r, j}$ then we get assumption (II) with $\gamma=\alpha, p_{ \pm}=p \pm q_{n}$ (and $\gamma\left(\gamma_{1}+\gamma_{2}\right) \leq \frac{1}{12}$ if $m, n$ are not too small). Here we use $M=n-m$.

To verify assumption (III) we use Corollary 6 . We obtain

$$
\sum_{\vec{v} \in R_{r}} \mu\left(C_{\vec{v}}\right) \leq C_{2} \alpha^{r-1} \sum_{j=2}^{r} \sum_{s=1}^{j-1}\left(\begin{array}{c}
j-1 \\
s-1
\end{array}\right)\left(\delta \mu\left(A_{n}\right)\right)^{j-s} \frac{\left(\tau \mu\left(A_{n}\right)\right)^{s}}{s !}\left(\begin{array}{c}
r-1 \\
j-1
\end{array}\right)\left(\alpha \mu\left(A_{m}\right)\right)^{r-j},
$$

where $\alpha=1+\phi(0)$. Hence condition (III) of Proposition 1 is satisfied with $\gamma_{1}=\delta \mu\left(A_{n}(x)\right)$, $\gamma_{2}=$ $\alpha \mu\left(A_{m}(x)\right)$ and $\beta=\mu\left(A_{n}(x)\right)$.

Let us note that this result applies to finite as well as infinite partitions $\mathcal{A}$. Since here we focus on the recurrence properties around periodic points we do not require the condition $\sum_{A \in \mathcal{A}}-\mu(A) \log \mu(A)<$ $\infty$ (which is necessary in order to get finite entropy or the theorem of Shannon-McMillan-Breiman).

Equilibrium states for Axiom A systems: Let us now assume that $\mu$ is an equilibrium state for a Hölder continuous function $f$ (with pressure zero) on an Axiom A space (shift space) which has the finite, generating partition $\mathcal{A}$ (see [3]). Then $\mu=h \nu$ where $h$ is a normalised eigenfunction for the largest eigenvalue of the transfer operator and $\nu$ is the associated eigenfunction. In particular $\nu$ is $e^{-f}$-conformal, i.e. if $T$ is one-to-one on a set $A$ then $\nu(T A)=\int_{A} e^{-f} d \nu(x)$. If we replace $f$ by $\tilde{f}=f+\log h-\log h \circ T$ then $\mu$ is $e^{-\tilde{f}}$-conformal. Thus, if $x$ is a periodic point with period $m$, then

$$
\mu\left(A_{n}(x)\right)=\mu\left(T^{m} A_{n+m}(x)\right)=\int_{A_{n+m}(x)} e^{-\tilde{f}^{m}(y)} d \mu(y)=\mu\left(A_{n+m}(x)\right) \tilde{q}_{n} e^{-\tilde{f}^{m}(x)},
$$

where $\tilde{q}_{n}$ is a number that can be estimated by

$$
\left|\log \tilde{q}_{n}\right| \leq \operatorname{var}_{n} \tilde{f}^{m} \leq \text { const. }\left(\operatorname{var}_{n} f+\operatorname{var}_{n+m} \log h+\operatorname{var}_{n} \log h\right) \leq \text { const. } \theta^{n},
$$

for some $\theta \in(0,1)$ (Hölder exponent). Hence

$$
\frac{\mu\left(A_{n+m}(x)\right)}{\mu\left(A_{n}(x)\right)}=p+q_{n},
$$

where $p=e^{f^{m}(x)}$ and $q_{n}=p\left(\tilde{q}_{n}-1\right)$ can be estimated by $\left|q_{n}\right| \leq p \theta^{n}$ const.. In particular the limit $\lim _{n \rightarrow \infty} \frac{\mu\left(A_{n+m}(x)\right)}{\mu\left(A_{n}(x)\right)}$ exists and equals $p$. It is known that $\mu$ is $\phi$-mixing where $\phi(k)=\rho^{k}$ for some $\rho \in(\theta, 1)$. Let us now apply Proposition 2 and in order to minimise the term $\epsilon_{n}=C_{1}\left(p^{\frac{n}{m}}+q_{n}+\rho^{\delta_{n}}\right)$ we choose $\delta_{n}=\frac{\log \mu\left(A_{n}(x)\right)}{\log \rho}$. Then $\epsilon_{n} \leq$ const. $\left(p^{\frac{n}{m}}+n \mu\left(A_{n}(x)\right)\right)$ (again $\left.M=n-m\right)$. 
Corollary 9 Let $\mu$ be an equilibrium state for a Hölder continuous function on an Axiom A system. Then there exists a constant $C_{5}$ so that for all periodic points $x, t>0$ and $r=0,1, \ldots$ one has ( $p$ is as above):

$$
\left|\mathbb{P}\left(\zeta_{n}^{t}=r\right)-e^{-t} P_{r}\right| \leq C_{5} \mu\left(A_{n}\right)\left|\log \mu\left(A_{n}(x)\right)\right| t^{r-1} \frac{e^{2 r}}{r !}+C_{5}\left(p^{\frac{n}{m}}+n \mu\left(A_{n}(x)\right)\right)\left\{\begin{array}{ll}
\frac{t^{r}}{r !} e^{2 r+\frac{5}{2} t} & \text { if } t>\frac{1}{2} p r \\
(2 p)^{r} e^{t \frac{1+2 p}{1-4 p}} & \text { if } t \leq \frac{1}{2} p r
\end{array} .\right.
$$

Algebraically $\phi$-mixing systems. If we assume that $\mu$ is $\phi$-mixing (with respect to the partition $\mathcal{A}$ ) where $\phi(k)=\mathcal{O}\left(k^{-\kappa}\right)$ for some $\kappa>0$, then let us note that

$$
p+q_{n}=\frac{\mu\left(A_{n+m}(x)\right)}{\mu\left(A_{n}(x)\right)} \leq \frac{(1+\phi(0)) \mu\left(A_{n}(x)\right) \mu\left(A_{m}(x)\right)}{\mu\left(A_{n}(x)\right)} \leq c_{1} \mu\left(A_{m}(x)\right)
$$

implies the very rough estimate $q_{n} \leq \mu\left(A_{m}(x)\right)$. With $\delta_{n}=\mu\left(A_{m}(x)\right)^{-\frac{1}{\kappa}}$ one now obtains $(n>>m)$

$$
\epsilon_{n} \leq c_{2}\left(p^{\frac{n}{m}}+\mu\left(A_{m}(x)\right)+\delta^{-\kappa}\right) \leq c_{3} \mu\left(A_{m}(x)\right) .
$$

Corollary 10 Let $\mu$ is $\phi$-mixing and $\phi(k) \sim k^{-\kappa}$ for some $\kappa>0$. Then there exists a constant $C_{6}$ so that for all periodic points $x, t>0$ and $r=0,1, \ldots$ one has ( $p$ is as above):

$$
\left|\mathbb{P}\left(\zeta_{n}^{t}=r\right)-e^{-t} P_{r}\right| \leq C_{6} \mu\left(A_{n}\right)\left|\log \mu\left(A_{n}(x)\right)\right| t^{r-1} \frac{e^{2 r}}{r !}+C_{6} n \mu\left(A_{m}(x)\right)\left\{\begin{array}{ll}
\frac{t^{r}}{r !} e^{2 r+\frac{5}{2} t} & \text { if } t>\frac{1}{2} p r \\
(2 p)^{r} e^{t \frac{1+2 p}{1-4 p}} & \text { if } t \leq \frac{1}{2} p r
\end{array} .\right.
$$

\subsection{Example}

In [19, 20] it has been shown that for ergodic systems every possible distribution can be realised for entry and return times of ergodic systems if the sequence of sets is suitably chosen. Naturally all settings in which the limiting distributions are shown to be exponential or Poissonian (in the case of higher returns) have to assume that the target set is a cylinder set (or a topological ball as in 25, 11). Here we show that even if we take cylinder sets then there are points which do not have a limiting distribution at all.

For simplicity's sake let $\Sigma$ be the full two shift with symbols 0,1 on which we put the Bernoulli measure with weights $w, 1-w>0\left(w \neq \frac{1}{2}\right)$. Let $y=0^{\infty}$ and $z=1^{\infty}$ be the two fixed points under the shift transformation $\sigma$. They have periods $m_{1}=m_{2}=1$. The entry times at $y, z$ are compound Poissonian with the $p$-weights $p_{1}=w$ and $p_{2}=1-w$. Put $\varepsilon=\frac{1}{3}\left|p_{1}-p_{2}\right|$ and we will now produce a point $x$ so that the return times distribution up to some order $r_{0}$ oscillates between the two compound Poisson distributions. Choose $n_{1}$ so that the cylinder $A_{n_{1}}(y)=A_{n_{1}}\left(0^{n_{1}}\right)$ has the distribution

$$
\left|\mathbb{P}\left(\zeta_{n_{1}}^{t}=r\right)-e^{-t} P_{r}\left(t, p_{1}\right)\right|<\frac{\varepsilon}{3}
$$

for $t \leq t_{0}$ and $r=1, \ldots, r_{0}$ for some $t_{0}>0$. Now we choose $n_{2}>n_{1}$ so that for the cylinder $A_{n_{2}}\left(0^{n_{1}} 1^{n_{2}-n_{1}}\right)$ one has

$$
\left|\mathbb{P}\left(\zeta_{n_{2}}^{t}=r\right)-e^{-t} P_{r}\left(t, p_{2}\right)\right|<\frac{\varepsilon}{3}
$$

for $t \leq t_{0}$ and $r=1, \ldots, r_{0}$. This can be done because the limiting distribution is invariant under the shift $\sigma$ (i.e. the limiting distribution of the cylinder $A_{n_{2}}\left(0^{n_{1}} 1^{n_{2}-n_{1}}\right)$ as $n_{2} \rightarrow \infty$ is equal to the limiting distribution of the cylinder $A_{n}\left(1^{\infty}\right)$ as $\left.n \rightarrow \infty\right)$. Continuing in this way we find a sequence of integers $n_{1}, n_{2}, n_{3}, \ldots$ so that the distribution of $\zeta_{n_{j}}^{t}$ alternates within an error of $\frac{\varepsilon}{3}$ between the distribution $e^{-t} P_{r}\left(t, p_{1}\right)$ (for odd $j$ ) and $e^{-t} P_{r}\left(t, p_{2}\right)$ (for even $j$ ) for $t \leq t_{0}$ and $r \leq r_{0}$. Hence the point $x=\bigcap_{j} A_{n_{j}}\left(0^{n_{1}} 1^{n_{2}-n_{1}} \cdots *^{n_{j}-n_{j-1}-\cdots-n_{1}}\right)$ ( $*$ is 0 is $j$ is odd and 1 if $j$ is even) has no limiting distribution.

Naturally, this construction can be carried out in all $\phi$-mixing systems. Instead of two fixed points one can also take any finite number of periodic points and then construct a point which takes turns visiting all of those so that at each visit it stays long enough so that its return time distribution gets arbitrarily close to the return time distribution of the periodic orbit it visits. 


\section{Return times}

Instead of looking at the probability of a randomly chosen point in the space $\Omega$ to enter a given set $A$, here we look at the statistics with which points within $A$ return to $A$ again. In the case of the first entry and return times, these two distributions have for general ergodic systems been linked in [12]. Higher order entry and return times have been related in [4]. It turns out that these distributions are the same only if the first return time is exponential. Similarly, the number of entry and return times have the same distribution if it is Poissonian. However, near periodic orbits we get for the return times a distribution which is very similar, namely it is in the limit given by the following compound Poisson distribution.

Let $p \in(0,1)$. If we define

$$
\hat{P}_{r}(t, p)=\sum_{j=0}^{r} p^{r-j}(1-p)^{j+1} \frac{t^{j}}{j !}\left(\begin{array}{l}
r \\
j
\end{array}\right)
$$

for $r=1,2, \ldots$ and $\hat{P}_{0}=1-p$ then the generating function for the probabilities $e^{-t} \hat{P}_{r}$ is

$$
\hat{g}_{p}(z)=e^{-t} \sum_{r=0}^{\infty} z^{r} \hat{P}_{r}=\frac{1-p}{1-z p} e^{t \frac{z-1}{1-p z}}
$$

The mean of this distribution is $\frac{t+p}{1-p}$ and the variance is $\frac{t+t p+p}{(1-p)^{2}}$. Again note that if $p=0$ then we get the Poisson terms $e^{-t} \hat{P}_{r}(t, 0)=e^{-t} \frac{t^{r}}{r !}$ and the generating function $e^{t(z-1)}$ which is analytic in the entire plane whereas for $p>0$ the generating function $\hat{g}_{p}(z)$ has an essential singularity at $\frac{1}{p}$. The expansion at $z_{0}=1$ yields $\hat{g}_{p}(z)=\sum_{k=0}^{\infty}(z-1)^{k} \hat{Q}_{k}$ where

$$
\hat{Q}_{k}(t, p)=\frac{1}{(1-p)^{k}} \sum_{j=0}^{k} p^{k-j} \frac{t^{j}}{j !}\left(\begin{array}{c}
k \\
j
\end{array}\right)
$$

$\left(\hat{Q}_{0}=1\right)$ are the factorial moments.

For a set $A$ let us now define the random variable $\hat{\zeta}_{A}=\chi_{A} \sum_{j=1}^{\tau_{n}} \chi_{A} \circ T^{j}$ and put $\hat{\zeta}_{n}^{t}=\hat{\zeta}_{A_{n}(x)}$ where $t=(1-p) \tau_{n} \mu\left(A_{n}(x)\right)$; we also denote with $\mu_{n}$ the conditional measure to the cylinder $A_{n}(x)$. In a similar way we can now prove the following result.

Theorem 11 Let $(\mu, \Omega)$ be a $\phi$-mixing measure with partition $\mathcal{A}, x$ a periodic point with period $m$ and $p$ and $q_{n}$ as above.

Then there exists a constant $C_{7}$ so that for every $\delta>0$ and every $t>0$ one has

$$
\left|\mathbb{P}\left(\hat{\zeta}_{n}^{t}=r \mid A_{n}\right)-e^{-t} \hat{P}_{r}\right| \leq C_{7} n \delta \mu\left(A_{n}\right) t^{r-1} \frac{e^{2 r}}{r !}+C_{7} n\left(p^{\frac{n}{m}}+q_{n}+\phi(\delta)\right)\left\{\begin{array}{ll}
\frac{t^{r}}{r !} e^{2 r+\frac{5}{2} t} & \text { if } t>\frac{1}{2} p r \\
(2 p)^{r} e^{t \frac{1+2 p}{1-4 p}} & \text { if } t \leq \frac{1}{2} p r
\end{array},\right.
$$

where $\tau_{n}=\frac{t}{(1-p) \mu\left(A_{n}(x)\right)}$.

If we compare these error terms to the ones for the entry times, we notice the additional factor $n$ which comes from satisfying the condition (I) of Proposition 1 (cf. [13]).

Let us note that for $r=0$ this result has previously been obtained by Hirata [15] for equilibrium states for Hölder continuous function on Axiom A systems. Here however we also get error estimates:

$$
\left|\mathbb{P}\left(\hat{\zeta}_{n}^{t}=0 \mid A_{n}\right)-(1-p) e^{-t}\right| \leq C_{6}\left(p^{\frac{n}{m}}+n \mu\left(A_{n}(x)\right)\right)
$$

Note that if $p>0$ then $\hat{P}_{0}(0, p)=1-p$ is strictly less than one and $\hat{P}_{r}(0, p)=p^{r}(1-p)$ for $r \geq 1$. There is a point mass at $t=0$ which corresponds to immediate returns within the neighborhood of the periodic point. These are clearly geometrically distributed. 
Remark: By adapting a recent remark of Chamoître and Kupsa [4, we proved in [14] under the condition of the existence of the asymptotic distribution of successive return times that the asymptotic distributions for the entry and return times are related by the formula $(k=1, \ldots)$

$$
D_{k}(t)=\int_{0}^{t}\left(\hat{D}_{k-1}(s)-\hat{D}_{k}(x)\right) d s
$$

where $D_{k}(t)$ is the limiting distribution $\mathbb{P}\left(\zeta_{n}^{t}=k\right)$ as $n \rightarrow \infty$, and $\hat{D}_{k}(t)=\lim _{n \rightarrow \infty} \mathbb{P}\left(\hat{\zeta}_{n}^{t}=k\right)$.

\section{$5 \quad$ Rational Maps}

Let $T$ be a rational map of degree at least 2 and $J$ its Julia set. Assume that we executed appropriate branch cuts on the Riemann sphere so that we can define univalent inverse branches $S_{n}$ of $T^{n}$ on $J$ for all $n \geq 1$. Put $\mathcal{A}^{n}=\left\{\varphi(J): \varphi \in S_{n}\right\}$ (n-cylinders). Note that the diameters of the elements in $\mathcal{A}^{n}$ go to zero as $n \rightarrow \infty$. Moreover, $\mathcal{A}^{n}$ is not the join of a partition, yet they have all the properties we require.

Let $f$ be a Hölder continuous function on $J$ so that $P(f)>\sup f(P(f)$ is the pressure of $f)$, let $\mu$ be its unique equilibrium state on $J$ and $\zeta_{n}=\sum_{j=1}^{\tau_{n}} \chi_{A_{n}} \circ T^{-j}$ the 'counting function' which measures the number of times a given point returns to the $n$-cylinder $A_{n}$ within the normalised time $\tau_{n}=\left[t / \mu\left(A_{n}\right)\right]$. Although $\mu$ is not a Gibbs measure we showed in [13. that for almost every $x$

$$
\mathbb{P}\left(\zeta_{n}=r\right) \rightarrow \frac{t^{r}}{r !} e^{-t}
$$

as $n \rightarrow \infty$.

Theorem 12 Let $T$ be a rational map of degree $\geq 2$ and $\mu$ an equilibrium state for Hölder continuous $f($ with $P(f)>\sup f)$.

Then there exists a $\tilde{\rho} \in(0,1)$ and $C_{8}$ so that for every periodic point $x \in J$ the return times are approximately compound Poissonian with the following error terms:

$$
\left|\mathbb{P}\left(\zeta_{n}^{t}=r\right)-e^{-t} P_{r}\right| \leq C_{8} \tilde{\rho}^{n} t^{r-1} \frac{e^{3 r}}{r !}+C_{8} \tilde{\rho}^{\frac{n}{m}}\left\{\begin{array}{ll}
\frac{t^{r}}{r !} \sqrt{r} & \text { if } t>\frac{1}{2} p r \\
(2 t r)^{r} e^{\frac{t}{p} \frac{1}{1-2 p}} & \text { if } t \leq \frac{1}{2} p r
\end{array},\right.
$$

where $p=e^{-f^{m}(x)-m P(f)}$ and $m$ is the minimal period of $x$.

The univalent inverse branches $S_{n}$ of $T^{n}$ (with appropriate branch cuts) split into two categories, namely the uniformly exponentially contracting inverse branches $S_{n}^{\prime}$ and the remaining $S_{n}^{\prime \prime}=S_{n} \backslash S_{n}^{\prime}$ for which do not contract uniformly. In [1] we showed the following result:

Lemma 13 (11] Lemma 9) Let $\eta \in(0,1)$. Then there exists a constant $v>0$ so that for all $r \geq 1$ and $\vec{v}=\left(v_{1}, v_{2}, \ldots, v_{r}\right) \in G_{r}$ satisfying $\min _{j}\left(v_{j+1}-v_{j}\right) \geq(1+v) n\left(\right.$ clearly $\left.r<\frac{\tau_{n}}{(1+v) n}\right)$ :

$$
\left|\frac{\mu\left(\bigcap_{j=1}^{r} T^{-v_{j}} W_{j}\right)}{\prod_{j=1}^{r} \mu\left(W_{j}\right)}-1\right| \leq \eta^{n},
$$

for all sets $W_{1}, \ldots, W_{r}$ each of which is a union of atoms in $\mathcal{A}^{n}$ and for all large enough $n$.

Let us define the rare set $R_{r}$ : We put $R_{r}$ for the set all $\vec{v} \in G_{r}\left(\tau_{n}\right)$ for which $\min _{j}\left(v_{j+1}-v_{j}\right) \leq(1+q) n$.

Lemma 14 Let $x \in J$ be a periodic point with (minimal) period $m$. For all large enough $n$ one has \{rational.period that $A_{n}(x) \cap T^{-\ell} A_{n}(x) \neq \emptyset$ for $\ell<n / 2$ only if $\ell$ is a multiple of $m$.

Proof. Put $n=k m+n^{\prime}, 0 \leq n^{\prime}<n$, and $\phi=\psi^{k} \cdots \psi^{1} \phi^{n^{\prime}}$, where $\psi^{1}, \ldots, \psi^{k} \in S_{m}, \phi^{n^{\prime}} \in S_{n^{\prime}}$. Since $x \in A$ is periodic with period $m$ we get that $T^{i m} A \cap A \neq \emptyset$ and in particular $x \in T^{i m} A$ for all $i=1, \ldots, k$. Since the sets $\psi\left(J \cap \Omega_{m}\right)$ are all disjoint for different $i$, we obtain $\psi^{i}=\psi^{1}$ for all $i$. Put $\psi=\psi^{1}$ and we get $\phi=(\psi)^{k} \phi^{k}$ (with $\psi$ concatenated $k$ times). 
Now assume that $A \cap T^{-\ell} A \neq \emptyset$ for some $\ell<\frac{n}{2}$ which is not a multiple of $m$. Since for some $i$, $i m<\ell<(i+1) m$ and $T^{i m} A \cap T^{-\ell+i m} A \neq \emptyset$, we can assume that $\ell<m$. Suppose that there are arbitrarily large $n$ so that $\ell<m$ and $V=A \cap T^{-\ell} A \neq \emptyset$. Similarly as above we put $n=k^{\prime} \ell+n^{\prime \prime}$ $\left(0 \leq n^{\prime \prime}<\ell\right)$ and obtain that $\phi \in S_{n}$ decomposes as $\phi=(\tilde{\psi})^{k^{\prime}} \tilde{\phi}^{n^{\prime \prime}}$ where $\tilde{\psi} \in S_{\ell}, \tilde{\phi}^{n^{\prime \prime}} \in S_{n^{\prime \prime}}$.

Now since $(\tilde{\psi})^{k^{\prime}}\left(J \cap \Omega_{\ell}\right) \rightarrow x$ as $k^{\prime} \rightarrow \infty$, and $x$ is periodic with period $m$, we see that such $\ell<m$ cannot exist. Hence, for all $n$ large enough $T^{\ell} A \cap A \neq \emptyset$ and $\ell<\frac{n}{2}$ implies that $\ell$ is a multiple of the period $m$.

Proof of Theorem 12, We are going to verify the conditions of Proposition 1, Let $x \in J$ be periodic with minimal period $m$. Then

(I) holds by invariance of the measure $\beta=\mu\left(A_{\varphi}\right)$ for all $j$.

(II) Since $\mu=h \nu$ where $h$ is a Hölder continuous density and $\nu$ is $e^{-f}$-conformal we obtain as before that

$$
\mu\left(A_{n}(x)\right)=\mu\left(T^{m} A_{n+m}(x)\right)=\int_{A_{n+m}(x)} e^{-\tilde{f}^{m}(y)} d \mu(y)=\mu\left(A_{n+m}(x)\right) \tilde{q}_{n} e^{-\tilde{f}^{m}(x)},
$$

where we have used that fact that $\mu$ is $e^{-\tilde{f}}$-conformal with respect to the function $\tilde{f}=f+\log h-\log h \circ T$. The factor $\tilde{q}_{n}$ satisfies $\left|\log \tilde{q}_{n}\right| \leq \operatorname{var}_{n} \tilde{f}^{m} \leq$ const. $\theta^{n}$, for some $\theta \in(0,1)$. Hence

$$
\frac{\mu\left(A_{n+m}(x)\right)}{\mu\left(A_{n}(x)\right)}=p+q_{n},
$$

where $p=e^{f^{m}(x)}$ (independent of $n$ ) and the error term $q_{n}=p\left(\tilde{q}_{n}-1\right)$ is bounded as $\left|q_{n}\right| \leq c_{1} p \theta^{n}$ for a constant $c_{1}$ which is independent of the periodic point $x$.

(III) Here we use Lemma 5 . By Lemma 14] we can choose $M=[n / 2]$. Furthermore we set $\delta=(1+v) n$. According to Lemma 13 our separation function $f$ is given by $f(k)=(1+v) k$. Hence $n^{\prime}=[n /(1+v)]$ and $m^{\prime}=[m /(1+v)]$. Then $A_{n^{\prime}}$ is the $n^{\prime}$-cylinder that contains $A_{n}=A_{n}(x)$ and whose measure is $\mu\left(A_{n^{\prime}}\right) \leq \rho^{n /(1+v)}$. Similarly $A_{m^{\prime}}$ is the $m^{\prime}$-cylinder that contains $A_{m}(x)$ and and whose measure is $\mu\left(A_{m^{\prime}}\right) \leq \rho^{m /(1+v)}$. Let us choose $\tilde{\rho}<1$ so that $\tilde{\rho}>\max \left(\rho^{\frac{1}{1+v}}, \eta, \vartheta\right)$. Then (for all large enough $n$ )

$$
\sum_{\vec{v} \in R_{r}} \mu\left(C_{\vec{v}}\right) \leq C_{2} \gamma^{r-1} \sum_{j=2}^{r} \sum_{s=1}^{j-1}\left(\begin{array}{c}
j-1 \\
s-1
\end{array}\right) \gamma_{1}^{j-s} \frac{\beta^{s}}{s !}\left(\begin{array}{c}
r-1 \\
j-1
\end{array}\right) \gamma_{2}^{r-j},
$$

where $\gamma_{1}=\delta \mu\left(A_{n^{\prime}}\right) \leq \tilde{\rho}^{n}, \gamma_{2} \leq \alpha \mu\left(A_{m^{\prime}}\right) \leq \tilde{\rho}^{m}, \beta=\tau \mu\left(A_{n}\right)$ and by Lemma $13 \alpha=1+\eta^{\delta-n^{\prime}}$. Moreover, since $p_{+}=p+q_{n} \leq \tilde{\rho}^{m}, \frac{\delta}{\tau} \leq \tilde{\rho}^{n}, p_{+}-p_{-} \leq q_{n} \leq \rho^{n}$ and $\phi=\phi\left(\delta-n^{\prime}\right) \leq \eta^{\delta-n^{\prime}} \leq \tilde{\rho}^{n}$ one has

$$
p_{+}^{\frac{n}{m}}+p_{+}-p_{-}+\phi \leq c_{1} \tilde{\rho}^{\frac{n}{m}}
$$

for some $c_{1}$. The theorem now follows from Proposition 1 .

\section{References}

[1] M Abadi and A Galves: Inequalities for the occurence times of rare events in mixing processes. The state of the art; Markov Proc. Relat. Fields 7 (2001) 97-112

[2] A D Barbour, L H Y Chen and W L Loh: Compound Poisson approximation for nonnegative random variables via Stein's method; Ann. Probab. 20 (1992), 1843-1866

[3] R Bowen: Equilibrium States for Anosov Diffeomorphism; Springer Lecture Notes 470, Springer, New York/Berlin 1975

[4] V Chamoître and M Kupsa: k-limit laws of return and hitting times; Discrete and Continuous Dynamical Systems 15 (2006), 73-86

[5] Z Coelho: Asymptotic laws for symbolic dynamical processes: In Topics in Symbolic Dynamics and Applications, edited by F Blanchard, A Maass and A Nogueira, LMS Lecture Notes Series 279, Cambridge University Press 2000, 123-165. 
[6] P Collet, A Galves and B Schmitt: Fluctuations of repetition times for Gibbsian sources; Nonlinearity 12 (1999) 1225-1237

[7] L H Y Chen and M Roos: Compound Poisson approximation for unbounded function on a group, with application to large deviations; Prob. Th. \& Rel. Fields 103 (1995) 515-528

[8] M Denker: Remarks on weak limit laws for fractal sets; Progress in Probability Vol. 37, Birkhäuser 1995, 167-178

[9] W Feller: An Introduction to Probability Theory and Its Applications; Wiley 1950

[10] A Galves and B Schmitt: Inequalities for hitting times in mixing dynamical systems; Random and Computational Dynamics 1997

[11] N Haydn: Statistical properties of equilibrium states for rational maps; Ergod. Th. \& Dynam. Syst. 20 (2000), 1371-1390

[12] N Haydn, Y Lacroix and S Vaienti: Hitting and Return Times in Ergodic Dynamical Systems: Ann. of Probab. 33 (2005), 2043-2050

[13] N Haydn and S Vaienti: The limiting distribution and error terms for return times of dynamical systems; Disc. Cont. Dyn. Syst. 10 (2004) 589-616

[14] N Haydn, E Lunedei and S Vaienti: Averaged Number of Visits; Chaos 17 (2007), 033119

[15] M Hirata: Poisson law for Axiom A diffeomorphisms; Ergod. Th. \& Dynam. Syst. 13 (1993), $533-556$

[16] M Hirata: Poisson law for the dynamical systems with the "self-mixing" conditions; Dynamical Systems and Chaos, Vol. 1 (Worlds Sci. Publishing, River Edge, New York (1995), 87-96

[17] M Hirata, B Saussol and S Vaienti: Statistics of return times: a general framework and new applications. Commun. Math. Phys. 206 (1999), 33-55

[18] N L Johnson, S Kotz and A W Kemp: Univariate Discrete Distributions, 3rd ed., Wiley Series in Probability and Mathematical Statistics: Applied Probability and Statistics, John Wiley \& Sons, New York, 2005.

[19] M Kupsa and Y Lacroix: Asymptotics for hitting times, Ann. of Probab. 33(3) (2005), 610-614

[20] Y Lacroix: Possible limit laws for entrance times of an ergodic aperiodic dynamical system; Israel J. Math. 132 (2002), 253-264

[21] L D Minkova: The Pólya-Aeppli process and ruin problems; J. Appl. Math. Stoch. Anal. 2004, $221-234$

[22] F Paccaut: Propriétés Statistiques de Systèmes Dynamiques Non Markovian; PhD Thesis Dijon 2000

[23] F Pène: Rates of Convergence in the CLT for Two-Dimensional Dispersive Billiards; Commun. Math. Phys. 225 (2002), 91-119

[24] W Philipp and W Stout: Almost sure invariance principles for partial sums of weakly dependent random variables; AMS Memoirs 161 (1975)

[25] B Pitskel: Poisson law for Markov chains; Ergod. Th. \& Dynam. Syst. 11 (1991), 501-513

[26] B A Sevast'yanov: Poisson limit law for a scheme of sums of independent random variables; Th. Prob. Appl. 17 (1972), 695-699

[27] H Wang, M Tang and R Wang: A Poisson limit theorem for a strongly ergodic non-homogeneous Markov chain; submitted to J. Math. Analysis and Applications. 